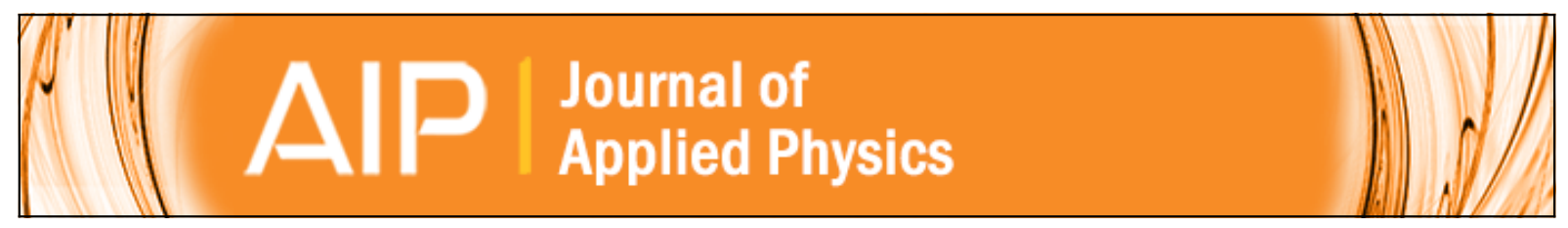

\title{
Magnetic force microscope study of domain wall structures in magnetite
}

Taras G. Pokhil and Bruce M. Moskowitz

Citation: Journal of Applied Physics 79, 6064 (1996); doi: 10.1063/1.362093

View online: http://dx.doi.org/10.1063/1.362093

View Table of Contents: http://scitation.aip.org/content/aip/journal/jap/79/8?ver=pdfcov

Published by the AIP Publishing

\section{Articles you may be interested in}

Study of $360^{\circ}$ domain walls in NiFe/NiO film by tip-sample interaction on magnetic force microscope J. Appl. Phys. 87, 6484 (2000); 10.1063/1.372745

Domain wall displacements in amorphous films and multilayers studied with a magnetic force microscope J. Appl. Phys. 81, 5035 (1997); 10.1063/1.364501

Localized micromagnetic perturbation of domain walls in magnetite using a magnetic force microscope Appl. Phys. Lett. 69, 3426 (1996); 10.1063/1.117281

Force gradient mapping of domain wall structures in magnetite (abstract)

J. Appl. Phys. 79, 6447 (1996); 10.1063/1.361968

Magnetic fine structure of domain walls in iron films observed with a magnetic force microscope J. Appl. Phys. 75, 5776 (1994); 10.1063/1.355613

\section{AlP $\mid$ APL Photonics}

APL Photonics is pleased to announce Benjamin Eggleton as its Editor-in-Chief

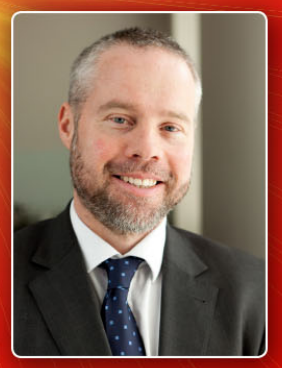




\title{
Magnetic force microscope study of domain wall structures in magnetite
}

\author{
Taras G. Pokhil and Bruce M. Moskowitz \\ Institute for Rock Magnetism, University of Minnesota, Minneapolis, Minnesota 55455
}

\begin{abstract}
Domain walls $(\mathrm{DW})$ in a small multidomain grain $(\approx 20 \mu \mathrm{m})$ of magnetite $\left(\mathrm{Fe}_{3} \mathrm{O}_{4}\right)$ exhibiting a planar domain pattern were studied using a magnetic force microscope (MFM). Most walls were subdivided with one or two Bloch lines and all walls displayed asymmetric MFM responses. Domain walls were observed to have small offsets either at the location of Bloch lines or at other locations without Bloch lines. The experimental data were described by a model in which (1) the easy axis of magnetization is not exactly parallel to the grain surface but is slightly inclined, and (2) there is also some plane dividing the grain in two parts with slightly different inclined easy axis directions. The inclined easy axis produces asymmetric spin distributions across the DW and wall offsets occur to reduce the surface magnetostatic energy of the wall. (C) 1996 American Institute of Physics. [S0021-8979(96)54908-7]
\end{abstract}

\section{INTRODUCTION}

The internal structure of domain walls (DW) between two domains with antiparallel magnetization has been intensively investigated both experimentally and theoretically. ${ }^{1-11}$ A variety of micromagnetic structures of domain walls in thin films and bulk materials have been observed and predicted including (1) domain walls with asymmetric spin distributions across the wall due to Néel-like structures called Néel caps terminating interior Block walls at surfaces, and (2) vortex-like spin distributions in Bloch lines separating opposite polarity wall segments. ${ }^{1-5}$ High spatial resolution imaging of micromagnetic structures using magnetic force microscopy provides experimental data on the structure of domain walls which can be used to test predictions of micromagnetic models. ${ }^{8-10}$

Magnetite is a ferrimagnetic oxide that occurs as a trace mineral in continental and ocean rocks. The magnetic memory in rocks of the ancient geomagnetic field is carried by the remanent magnetization of magnetite particles less than $50 \mu \mathrm{m}$ in size. ${ }^{12}$ The term pseudo-single domain (PSD) describes magnetic behavior that is intermediate between classical single domain (SD) and multidomain (MD) behavior and is usually attributed to particles containing just a few $(<10)$ domains. The physics of PSD behavior plays a central role in paleomagnetism because most magnetic oxide grains in rocks are too large to be in an equilibrium SD state $(<0.1$ $\mu \mathrm{m})$ and contain domains, yet can carry geologically stable remanence. In this paper we present results of a magnetic force microscope (MFM) study of domain wall structures in a small grain of magnetite containing a few domains.

\section{EXPERIMENTAL METHODS}

Small grains $(5-50 \mu \mathrm{m})$ of magnetite $\left(\mathrm{Fe}_{3} \mathrm{O}_{4}\right)$, randomly oriented and dispersed in nonmagnetic matrix, were produced by the glass-ceramic method. ${ }^{13}$ The sample was mounted in epoxy and mechanically polished with diamond compounds. Amorphous silica solution was used as a final polish to obtain a smooth surface and to reduce the strained surface layer produced during the initial mechanical polishing. ${ }^{14}$ The bulk coercive force of the sample was 15 Oe.
Magnetic force images were obtained with a Nanoscope III scanning probe microscope. The microscope was operated in the "tapping/lift" scanning mode, ${ }^{15}$ which combines constant interaction and constant height modes, to separate topographic and magnetic signals. The scanned probes were batch fabricated $\mathrm{Si}$ cantilevers with pyramidal tips coated with a CoCr film alloy. ${ }^{15}$ All MFM data shown in this paper were collected with the tip magnetized approximately perpendicular to the sample surface ( $z$ direction), making the MFM sensitive to the second derivative of the $z$ component of sample stray field. To exclude any influence of the MFM tip on the sample micromagnetic structure, images were taken with various tip-sample orientations and tip-sample separations. Under these experimental conditions we did not observe any noticeable modifications of micromagnetic features during MFM scanning. All MFM images presented in this paper were obtained with tip sample separation of $50 \mathrm{~nm}$ and tip vibration amplitude of $20-30 \mathrm{~nm}$. The drive frequency of cantilever was chosen above the resonance frequency of the cantilever near the point of maximum gradient of the cantilever resonance curve.

\section{RESULTS}

We concentrated our MFM study on a $20 \mu \mathrm{m}$ grain exhibiting a simple planar domain structure. Although the crystallographic orientation of the grain surface was unknown, the simple domain pattern suggested that the direction of magnetization within the domains was approximately parallel to the surface of the grain. Domain walls in this grain were approximately parallel to each other with domain spacing of 2-3 $\mu \mathrm{m}$ (Fig. 1). The FWHMs of the MFM response profiles across the walls were about $200 \mathrm{~nm}$ which is slightly wider than the theoretical Bloch wall width in bulk magnetite $(100-150 \mathrm{~nm}),{ }^{16}$ but similar to MFM results obtained for a large single crystal of magnetite. ${ }^{10}$ Surface broadening of the DW can result from the self-demagnetizing field acting on the DW near the grain surface resulting in, for instance, a Néel cap. However, some broadening of the MFM response is expected due to the integrated effect over the tip. Some walls were subdivided into alternating chirality segments separated by Bloch lines. The smallest observed distance between Bloch lines was $\approx 1 \mu \mathrm{m}$. 


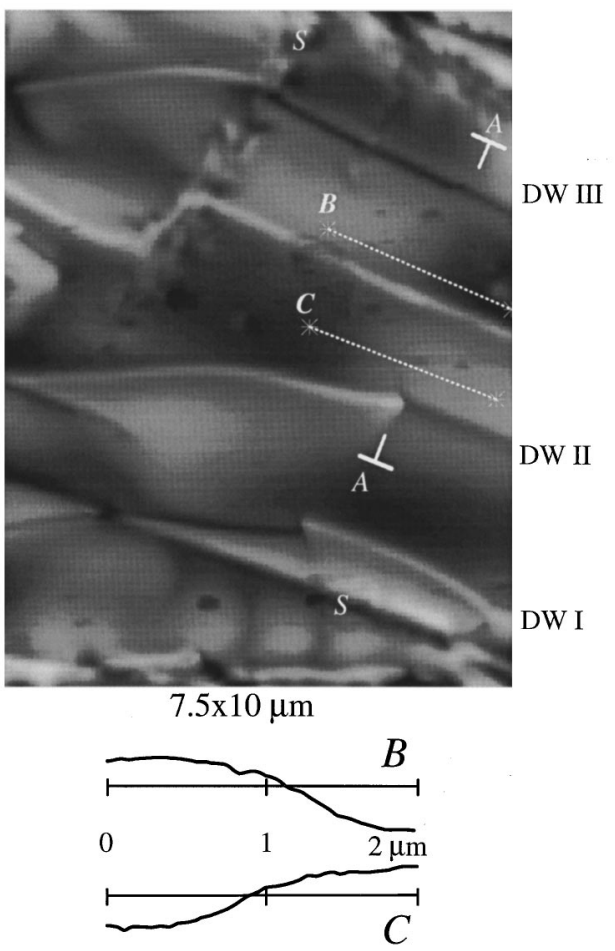

FIG. 1. MFM image of domain walls in a magnetite grain and MFM response profiles along the domains at lines $B$ and $C$. Features marked as $S$ are stray fields from scratches.

We found several interesting spin features associated with domain walls. Small offsets of the DW were observed in some walls at the location of Bloch lines [Fig. 2(c)], whereas other walls had offsets without an accompanying Bloch line [Fig. 2(a)]. The size of these offsets varied between 30 and $50 \mathrm{~nm}$. The MFM response profiles across these walls were asymmetric. Moreover, the MFM profiles $A$ taken on one side of DW offset point can be transformed into profiles $B$ taken on the opposite side of the offset using axially symmetric [Figs. 2(a) and 2(b)] or centrally symmetric transformations [Fig. 2(c)]. We also observed a change of the MFM response within the domains. For example, in Fig. 1, the MFM response changes when the MFM tip crosses line $A-A$ and the sign of the change is opposite in neighboring domains resulting in checkerboard contrast pattern. This is seen more clearly in Fig. 2 which shows higher-resolution image scans of the walls labeled DW I, DW II, and DW III in Fig. 1. Here, there is a change in the MFM response within the domains across the wall offset associated with DW III [Fig. 2(a)] and the Bloch line in DW II [Fig. 2(b)], but no change in MFM response associated with the offset at the location of the Bloch line in DW I [Fig. 2(c)].

\section{DISCUSSION}

To explain our experimental data (DW offsets and asymmetric MFM profiles), we proposed the following simple model depicted in Figs. 3 and 4. We assumed that (1) the easy axis of magnetization is not in the plane of the grain surface but is slightly inclined and that the direction of magnetization inside the domains is parallel to the easy axis; and (2) a plane intersecting the domain walls divides the grain in
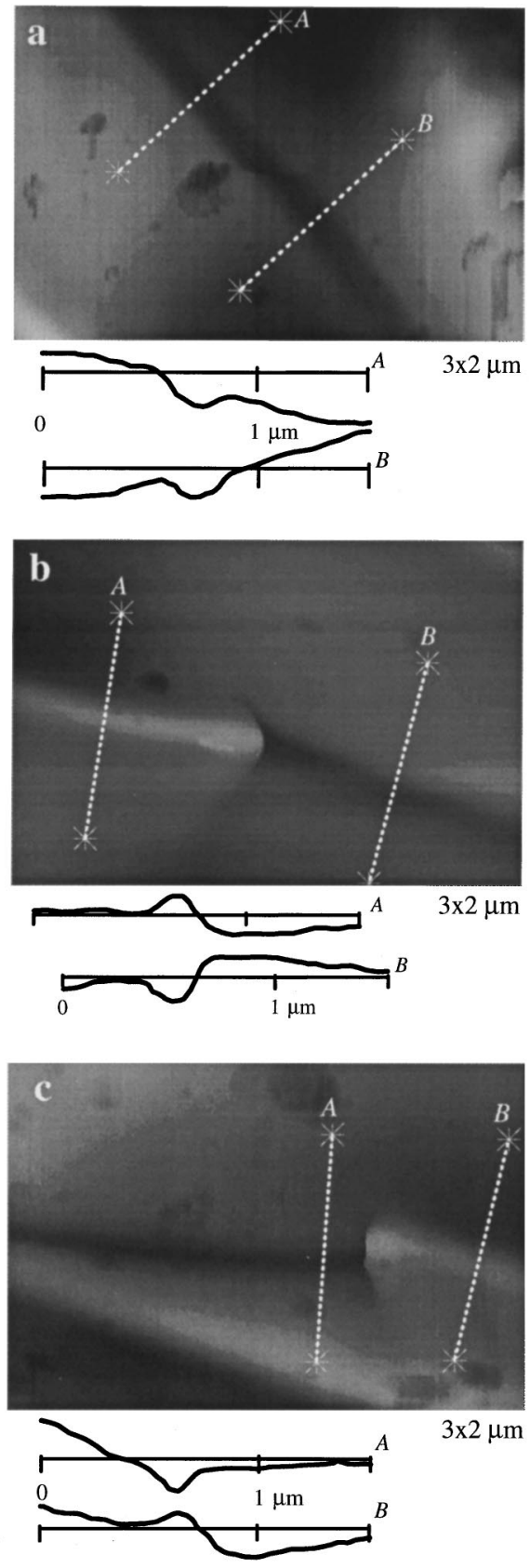

FIG. 2. MFM images of three walls in Fig. 1, DW I, DW II, and DW III and MFM response profiles across the domain walls at lines $A$ and $B$. The size of all images is $3 \times 2 \mu \mathrm{m}$. (a) DW without a Bloch line; easy axis of magnetization has different directions on different sides of the DW offset point, (b) DW with a Bloch line; easy axis has different directions on different sides of the Bloch line. (c) DW with a Bloch line; easy axis has the same direction on different sides of the Bloch lines.

two parts with slightly different inclined easy axis directions (Fig. 4). Under these conditions, the magnetization in each domain will have a small component perpendicular to the surface. Within the same domain, this component is directed out of (into) the surface on one side of the dividing plane and directed into (out of) the surface on the opposite side of the plane. The inclined easy axis relative to the sample surface produces the observed asymmetric spin distribution across the DW. This hypothesis is consistent with the observed change of MFM response within the domains (i.e., the 

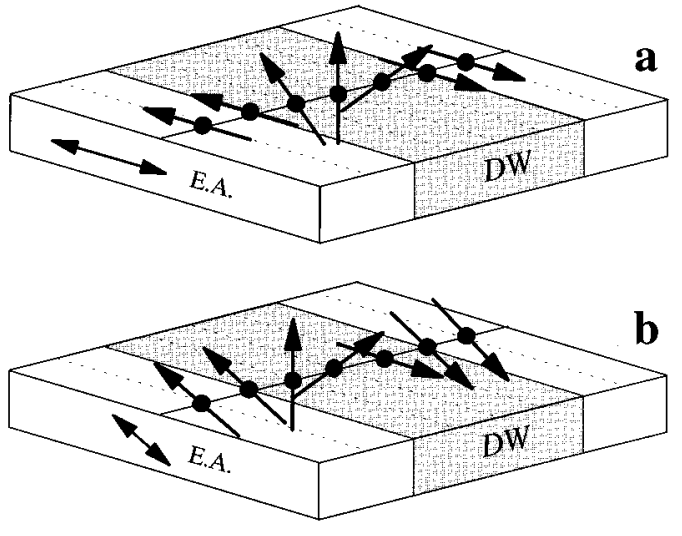

FIG. 3. Distribution of magnetic moments across a Bloch domain wall where (a) the easy axis is in the plane of the surface; and (b) the easy axis makes a small angle with the surface. The MFM response across the wall would be symmetric for (a) and asymmetric for (b).

checkerboard pattern in Fig. 1). Such a change of easy axis direction can result from residual surface stress, or from the formation of subgrain or twin boundaries during crystallization which divides the grain in two parts with slightly different crystallographic orientations.

Spin distributions across a Bloch wall when the easy axes are in plane or inclined are presented in Figs. 3(a) and 3(b), respectively. For the DW shown in Fig. 3(b), the area of the wall with magnetization perpendicular to the surface is shifted toward one side of the wall, while near the other side of the wall, there is an area in which the direction of magnetization is parallel to the surface. The magnetostatic energy of a DW crossing an area where the easy axis changes direction is reduced if the DW is offset and "in-plane" spins in the DW on both sides of this area follow the magnetization in the adjacent domains [Fig. 4(a)]. The stray field distribution above the DW on both sides of wall offset point would be asymmetric as shown in Fig. 4(a). These model field distributions are qualitatively similar to the MFM response profiles in Fig. 2(a). Similarly, it follows that (1) a DW offset is magnetostatically favorable at the location of Bloch line when the easy axis is inclined, but does not change its direction at the Bloch line [Fig. 4(c)]; and (2) no DW offset should occur at the location of a Bloch line if it happens to be located in the plane where the easy axis changes direction [Fig. 4(b)]. The MFM images of domain walls with Bloch lines in Figs. 2(b) and 2(c) agree with this simple interpretation. This model also explains the symmetry relationships exhibited by the MFM response profiles on opposite sides of offset points (Fig. 2). Using the model and measured wall offsets, the angle of the inclined magnetization within the domains with respect to the surface of the grain was calculated to be approximately $20^{\circ}$.

\section{ACKNOWLEDGMENTS}

We thank Sheryl Foss for useful discussions and critical reading of the manuscript and Matthew Dugas (Advanced

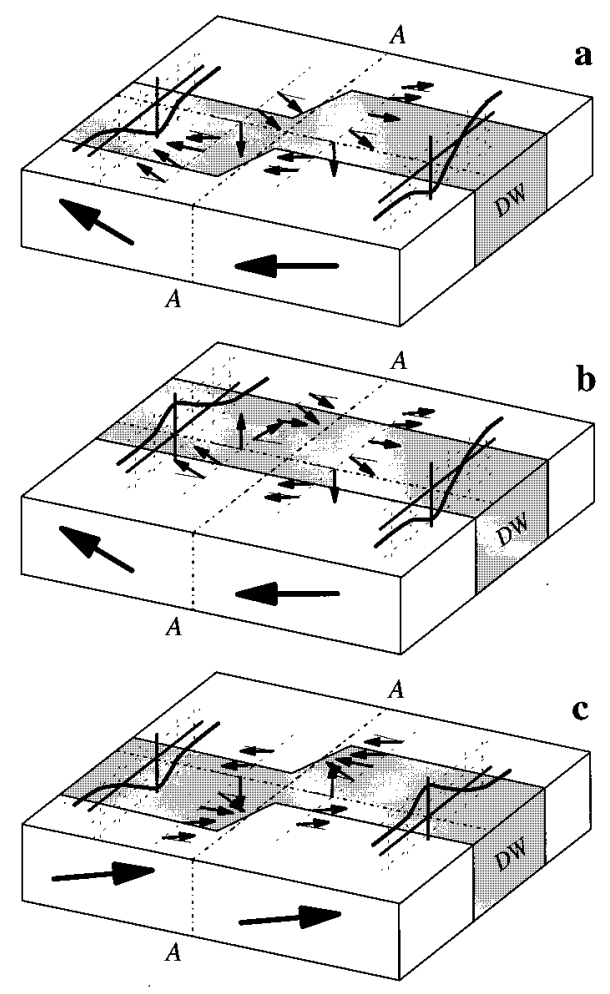

FIG. 4. Schematic drawing of an asymmetric Bloch DW. In (a) the DW has an offset but no Bloch line. The easy axis of magnetization has different directions on different sides of the DW offset point. In (b) the DW has a Bloch line but no offset. The easy axis has different directions on different sides of the Bloch line. In (c) the DW has both a Bloch line and an offset. The easy axis has the same direction on different sides of the Bloch line.

Research Corporation) for preparation of MFM tips with sputtered $\mathrm{CoCr}$ thin films. This work was partially supported by the NSF. This is contribution 9503 of the Institute for Rock Magnetism. The Institute for Rock Magnetism is supported by grants from Keck Foundation and NSF.

${ }^{1}$ A. E. LaBonte, J. Appl. Phys. 40, 2450 (1969)

${ }^{2}$ A. Hubert, Phys. Status Solidi 38, 699 (1970).

${ }^{3}$ A. Hubert, IEEE Trans. Magn. MAG-11, 1285 (1975).

${ }^{4}$ U. Hartmann, Phys. Status Solidi B 151, 289 (1989).

${ }^{5}$ M. R. Scheinfein, J. Unguris, R. J. Celotta, and D. T. Pierce, Phys. Rev. Lett. 63, 668 (1989).

${ }^{6}$ L. Schön and U. Buchenau, Intern. J. Magn. 3, 145 (1972).

${ }^{7}$ P. Trouilloud and J. Miltad, J. Magn. Magn. Mater. 66, 1934 (1987).

${ }^{8}$ U. Hartmann, Phys. Rev. B 40, 7421 (1989).

${ }^{9}$ W. Williams, V. Hoffman, F. Heiden, G. Göddenhenrich, and C. Heiden, Geophys. J. Int. III, 417 (1992).

${ }^{10}$ R. B. Proksch, S. Foss, and E. D. Dahlberg, IEEE Trans. Magn. MAG-30, 4467 (1994).

${ }^{11}$ M. R. Scheinfein, J. Unguris, D. T. Pierce, and R. J. Celotta, J. Appl. Phys. 67, 5932 (1990).

${ }^{12}$ D. J. Dunlop, Rep. Prog. Phys. 53, 707 (1990).

${ }^{13}$ H. U. Worm and H. Market, Phys. Earth Planet. Int. 46, 84 (1987).

${ }^{14}$ V. Hoffman, R. Schäfer, E. Apple, A. Hubert, and H. Suffel, J. Magn. Magn. Mater. 71, 90 (1987).

${ }^{15}$ Nanoscope III, Digital Instruments, Santa Barbara, CA.

${ }^{16}$ B. M. Moskowitz and S. K. Banerjee, IEEE Trans. Magn. MAG-15, 1241 (1979). 